EPJ Web of Conferences 27, 00010 (2012)

DOI: $10.1051 /$ epjconf/20122700010

(C) Owned by the authors, published by EDP Sciences, 2012

\title{
Prior for Structural Materials
}

\author{
D. Neudecker ${ }^{1, \text { a }}$, St. Gundacker ${ }^{2}$, H. Leeb ${ }^{1, b}$, Th. Srdinko ${ }^{1}$, and V. Wildpaner ${ }^{1}$ \\ 1 Atominstitut, Technische Universität Wien, Vienna, Austria \\ 2 CERN, Geneva, Switzerland
}

\begin{abstract}
The construction of a library containing prior cross sections and related covariance matrices for nine frequently measured reaction channels of 276 structural materials from 1 to $150 \mathrm{MeV}$ is presented. This library provides a starting point for future evaluations and reduces them to the analysis of experimental data. The prior cross sections were calculated using the nuclear reaction programs of TALYS1.0 and TALYS1.2 with the implemented global and local neutron and proton optical potential parameter sets. The prior covariance matrices contain contributions stemming from parameter uncertainties and model deficiencies. The associated procedures are discussed and an example evaluation with these prior cross sections and covariance matrices is shown for one specific isotope. These developments are of great importance with regard to the reliability of evaluations strongly based on model calculations. This is particularly true for the extension of the energy range as requested by the development of novel nuclear technologies.
\end{abstract}

\section{Introduction}

Current efforts in nuclear data evaluation are triggered by the development of novel nuclear technologies and safety requirements. There are two major demands, i.e. (1) the supply of reliable uncertainty estimates in the form of covariance matrices and (2) the extension of the energy range of the data files up to $150-200 \mathrm{MeV}$. Because of the scarcity of experimental data beyond $20 \mathrm{MeV}$ in most reaction channels such extended evaluated data sets rely heavily on nuclear models. Consequently, the determination of associated covariance matrices must account not only for the experimental uncertainties, but also for the limitations of the models employed.

A reliable uncertainty estimate of nuclear calculations must account at least for the limited knowledge of model parameters as well as for the deficiencies of the model. The former depends strongly on the admissible bounds of model parameters, which should be chosen reasonably, best on the basis of physics and mathematics constraints. Apart from this choice the calculation of corresponding covariance matrices is straightforward, albeit tedious. The estimate of model deficiencies is much more difficult and there exist only few procedures [1-3] for their determination.

A nuclear data evaluation aims at the best knowledge of cross sections combining the experimental information with the predictions of nuclear models in the Bayesian paradigm. The cross sections and associated covariance matrices generated by nuclear models represent the prior knowledge for the evaluation procedure.

In this contribution, we present a library with prior sets for 276 isotopes relevant for structural materials. The prior cross sections were calculated with programs of TALYS1.0 and TALYS1.2 [4] using implemented local or global optical potential model parameter sets [5]. The prior covariance matrices contain contributions from parameter uncer- tainties and model defects. Section 2 briefly summarizes the procedures. In order to show the feasibility of this library, an example evaluation with ${ }^{55} \mathrm{Mn}$ prior data is compared to an evaluation with a prior specifically made for this isotope, see Section 3. A summary and outlook is provided in Section 4.

\section{Generation of Prior Knowledge}

Prior cross sections and covariance matrices were calculated for nine frequently measured channels of 276 isotopes of structural materials. The library was constructed for angle-integrated cross sections of incoming neutrons. The nine channels are given in Table 1 and the isotopes provided over a great part of the periodic table - are shown in Fig. 1. Isotopes from ${ }^{24} \mathrm{Mg}$ to ${ }^{209} \mathrm{Bi}$ were considered in the library. These isotopes are within the validity range of the global and local optical potential by [5]. In addition, the employed models implemented in TALYS are not adequate for the other mass-values. Furthermore, only those isotopes were calculated for which at least three experimental data sets are available in EXFOR [6] for five channels of Table 1.

The prior cross sections and covariance matrices are valid for evaluations in an energy range from 1 to $150 \mathrm{MeV}$. Also an energy bin from $1.0 \mathrm{e}-05 \mathrm{eV}$ to $1 \mathrm{MeV}$ is supplied in order to conform to the ENDF-6 standard. However, the methods [1] employed to calculate this library have been developed for evaluations above the resonance region.

Table 1. List of channels available in the library.

\begin{tabular}{lllll}
\hline total & elastic & nonelastic & inelastic & \\
\hline$(n, 2 n)$ & $(n, 3 n)$ & $(n, \gamma)$ & $(n, p)$ & $(n, \alpha)$ \\
\hline
\end{tabular}

\footnotetext{
a e-mail: dn@kph.tuwien.ac.at
}

b e-mail: leeb@kph.tuwien.ac.at 


\begin{tabular}{|c|c|c|c|c|c|c|c|c|c|c|c|c|c|c|c|c|c|}
\hline & \begin{tabular}{|l|}
12 \\
$\mathrm{Mg}$ \\
$24-26$
\end{tabular} & & & & & & & & & & & $\begin{array}{l}13 \\
\text { Al } \\
26,27\end{array}$ & \begin{tabular}{|l|}
14 \\
${ }^{14}$ \\
$28-30$
\end{tabular} & 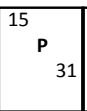 & \begin{tabular}{|r|}
16 \\
$\mathrm{~s}$ \\
$32-34$, \\
36
\end{tabular} & $\begin{array}{l}17 \\
\mathrm{Cl} \\
35-37\end{array}$ & \begin{tabular}{|r|}
18 \\
Ar \\
36,38 \\
40 \\
\end{tabular} \\
\hline \begin{tabular}{|l|}
37 \\
$\mathbf{R b}$ \\
85,87
\end{tabular} & \begin{tabular}{|c|}
38 \\
Sr \\
$84,86-$ \\
88
\end{tabular} & $\begin{array}{c}39 \\
Y \\
88,89\end{array}$ & \begin{tabular}{|c|}
40 \\
$\mathrm{Zr}$ \\
$88: 2: 96$, \\
\\
91
\end{tabular} & 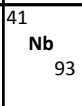 & \begin{tabular}{|l|}
42 \\
Mo \\
$92: 2: 100$ \\
95,97 \\
\end{tabular} & 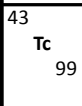 & \begin{tabular}{|l|}
\multicolumn{1}{|l|}{$\mathbf{R u}$} \\
$96: 2: 104$ \\
99,101
\end{tabular} & $\begin{array}{l}\text { Rh } \\
103\end{array}$ & \begin{tabular}{|c}
46 \\
$\mathbf{P d}$ \\
$102: 2: 10$ \\
, 105
\end{tabular} & $\begin{array}{r}47 \\
\mathrm{Ag} \\
107 \\
109\end{array}$ & \begin{tabular}{|c|}
48 \\
Cd \\
$106: 2: 16$ \\
111,113
\end{tabular} & \begin{tabular}{|l}
49 \\
In \\
113, \\
115
\end{tabular} & \begin{tabular}{|l|}
50 \\
Sn \\
112,14 \\
$20,22,24$
\end{tabular} & \begin{tabular}{|c|}
1 \\
Sb \\
121 \\
123
\end{tabular} & \begin{tabular}{|l|}
52 \\
Te \\
$120: 2: 30$ \\
23,25
\end{tabular} & $\begin{array}{l}3 \\
127, \\
129\end{array}$ & \begin{tabular}{|l|}
\multicolumn{1}{|l|}{$\mathbf{X}$} \\
$\mathbf{X e}$ \\
$124: 2: 36$ \\
29,31 \\
\end{tabular} \\
\hline \begin{tabular}{|r|}
55 \\
Cs \\
133 \\
135 \\
\end{tabular} & \begin{tabular}{|l|}
56 \\
Ba \\
$130: 2: 38$ \\
35,37 \\
\end{tabular} & 57-71 & \begin{tabular}{|l|}
72 \\
Hf \\
$174: 2: 80$ \\
77,79 \\
\end{tabular} & \begin{tabular}{|c}
73 \\
Ta \\
180, \\
181
\end{tabular} & \begin{tabular}{|r|}
74 \\
$W$ \\
$182: 84$ \\
186
\end{tabular} & \begin{tabular}{|r}
75 \\
$\operatorname{Re}$ \\
185 \\
187
\end{tabular} & \begin{tabular}{|l}
76 \\
Os \\
$184: 2: 92$ \\
187,89
\end{tabular} & \begin{tabular}{|l}
77 \\
Ir \\
191 \\
193
\end{tabular} & \begin{tabular}{|l}
8 \\
Pt \\
$190: 2:$ \\
98,95
\end{tabular} & $\begin{array}{r}79 \\
\text { Au } \\
197, \\
198\end{array}$ & \begin{tabular}{|l|}
80 \\
Hg \\
$196: 2: 04$ \\
199,201
\end{tabular} & \begin{tabular}{|l|}
81 \\
TI \\
203, \\
205
\end{tabular} & \begin{tabular}{|r|}
82 \\
$\mathrm{~Pb}$ \\
$204: 2: 8$, \\
207 \\
\end{tabular} & \begin{tabular}{|c|}
83 \\
$\quad$ Bi \\
209
\end{tabular} & & & \\
\hline
\end{tabular}

\begin{tabular}{|c|c|c|c|c|c|c|c|c|c|c|c|c|c|c|c|}
\hline Lanthanides: & \begin{tabular}{|c|}
57 \\
La \\
138, \\
139
\end{tabular} & \begin{tabular}{|l|}
58 \\
$\mathrm{Ce}$ \\
$136: 2:$ \\
$\quad 142$
\end{tabular} & \begin{tabular}{|l|l|}
59 & \\
& $\operatorname{Pr}$ \\
& \\
& 141
\end{tabular} & \begin{tabular}{|l|}
60 \\
Nd \\
$142-46$ \\
148150
\end{tabular} & \begin{tabular}{|l}
61 \\
Pm \\
147
\end{tabular} & $\begin{array}{c}62 \\
\text { Sm } \\
147-52 \\
144-54\end{array}$ & \begin{tabular}{|l|l|}
63 & \\
& Eu \\
& 151 \\
& 153 \\
\end{tabular} & $\begin{array}{l}64 \\
\text { Gd } \\
154-58\end{array}$ & $\begin{array}{l}65 \\
\quad \text { Tb } \\
159\end{array}$ & \begin{tabular}{|l|}
66 \\
Dy \\
$156: 2: 64$ \\
$161: 63$
\end{tabular} & $\begin{array}{|ll|}67 & \\
& \text { Ho } \\
& 156\end{array}$ & \begin{tabular}{|l|}
68 \\
Er \\
$162: 2:$ \\
70.67
\end{tabular} & \begin{tabular}{|l|}
69 \\
Tm \\
169, \\
$\quad 171$
\end{tabular} & \begin{tabular}{|c|}
70 \\
Yb \\
$168: 2: 76$ \\
7173
\end{tabular} & $\begin{array}{l}1 \\
\text { Lu } \\
175, \\
176\end{array}$ \\
\hline
\end{tabular}

Fig. 1. The isotopes available in the library are displayed. ' $162: 2: 70$ ' is equivalent to ' $162,164,166,168,170$ '.

The prior covariance matrices contain contributions of parameter uncertainties as well as model defects. Covariances are supplied for all pairs of energy bins of the same channel as well as between different channels. Covariances between different isotopes are not supplied in the current version.

The prior cross sections and covariance matrices are given in ENDF-6 format in order to comply with the standard format of nuclear data evaluation. The cross sections are given in $\mathrm{MF}=3$ files and the covariance matrices in $\mathrm{MF}=33$ files. A general description of the generation procedure and details for the specific isotope are given in $\mathrm{MF}=1$ files.

\subsection{Theoretical Cross Sections $\sigma_{t h}$}

The quality of the evaluated data will be high in general, if the prior gives a fair description of the experiments. Therefore, we used the well-established and tested local and global parameter sets for the neutron and proton optical model potential by Koning and Delaroche [5]. The local neutron optical potential parameters were obtained by a method based on $\chi^{2}$ optimization and simulated annealing and using neutron elastic scattering cross sections, total cross section and proton elastic scattering data [5]. From the local parameters of single isotopes, a global parameterization for the neutron and proton optical model parameters was constructed. Only for the isotopes ${ }^{27} \mathrm{Al},{ }^{40} \mathrm{Ca},{ }^{56} \mathrm{Fe},{ }^{58} \mathrm{Ni},{ }^{89} \mathrm{Y}$, ${ }^{90} \mathrm{Zr},{ }^{93} \mathrm{Nb},{ }^{197} \mathrm{Au},{ }^{208} \mathrm{~Pb}$ and ${ }^{209} \mathrm{Bi}$, experimental total cross sections were considered in the optimization procedure. In order to avoid double counting of experimental data during a future evaluation with a local prior of one of these isotopes, only experimental total cross section data should be used which are not listed in Table 2 of [5]. If global prior cross sections and covariance matrices are used in a future evaluation, no special care needs to be taken regarding possible double counting of experimental data.

If a local parameterization of the optical potential was not available, the global one was used. For the level density and the charged particle potential parameters the default values of TALYS [4] were taken. For each isotope, the prior cross sections and associated uncertainties were calculated using TALYS1.0 and TALYS1.2 for local and global parameter sets, resulting in total in four priors for each isotope.

\subsection{Parameter Uncertainty Covariance Matrices $A_{0}^{P U}$}

The covariance matrices $A_{0}^{P U}$ account for the limited knowledge of the initial model parameters $\underline{a}_{0}$ described in Section 2.1. They are estimated by

$$
A_{0}^{P U}\left(E_{j}, E_{k}\right)=\frac{1}{I} \sum_{i=1}^{I}\left(\sigma_{j}\left(\underline{a}_{i}\right)-\sigma_{t h, j}\right)\left(\sigma_{k}\left(\underline{a}_{i}\right)-\sigma_{t h, k}\right)
$$

following the methods in [7]. The theoretical cross sections $\sigma_{t h}=\sigma\left(\underline{a}_{0}\right)$ are calculated according to Section 2.1. The cross sections $\sigma_{j}$ and $\sigma_{k}$ are calculated by TALYS [4] using parameter vectors $\underline{a}_{i}$. These are randomly selected following a uniform distribution within defined parameter boundaries $\underline{a}^{<}$and $\underline{a}^{>}$. The parameters are assumed to be independent from each other. For each isotope 3000 parameter vectors are selected each for global and local TALYS1.2 priors and 1500 parameter vectors are selected for global and local TALYS1.0 priors. Each parameter vector corresponds to a call of TALYS, resulting in a total of about 300 000CPU hours on the Vienna Scientific Cluster to compute $A_{0}^{P U}$ for all isotopes in Fig. 1.

However, the main problem were not the time-intensive calculations, but to establish reasonable parameter boundaries for each parameter of all isotopes. In [8], an example showed that unphysically small parameter boundaries can result in deficient output even if sufficient experimental data are included. Otherwise if the parameter boundaries are chosen much too large, the evaluated uncertainties might also be too large and therefore of little use. Hence, special care was taken to establish the parameter boundaries on a physical basis if possible, and to limit the boundaries to a reasonable size. In the following, it is shown by means of two parameters, i.e. $R_{V}$ and $d_{2}$, how the associated parameter boundaries were established on a physical and 'phenomenological' basis. 
Parameter Boundaries for $R_{V}$ The parameter $R_{V}$ is the radius of the real volume-central contribution $\mathscr{V}_{V}$ to the phenomenological neutron optical potential. This parameter appears in the radial Woods-Saxon form of $\mathscr{V}_{V}$,

$$
f\left(r, R_{V}, a_{V}\right)=\left(1+\exp \left\{\frac{r-R_{V}}{a_{V}}\right\}\right)^{-1},
$$

with the diffuseness parameter $a_{V}$. The optical potential $\mathscr{V}_{V}$ consists of a product of $f\left(r, R_{V}, a_{V}\right)$ and an energydependent part which is of no further interest for this consideration.

In order to estimate physical parameter boundaries, we calculate a mean square radius $\left\langle r_{o p}^{2}\right\rangle$ of $\mathscr{V}_{V}$ by

$$
\left\langle r_{o p}^{2}\right\rangle=\frac{\int r^{2} \mathscr{V}_{V} \mathrm{~d}^{3} r}{\int \mathscr{V}_{V} \mathrm{~d}^{3} r}=\frac{\int_{0}^{\infty} r^{4} f\left(r, R_{V}, a_{V}\right) \mathrm{d} r}{\int_{0}^{\infty} r^{2} f\left(r, R_{V}, a_{V}\right) \mathrm{d} r} .
$$

This radius of the interaction should be larger than, or at least equal to, the mean square charge radius of the nucleus $\left\langle r_{n}^{2}\right\rangle$. The margin of $\left\langle r_{o p}^{2}\right\rangle$ is related to the range of the strong interaction, i.e. $r_{f}=1.4 \mathrm{fm}$. Thus, we obtain the following condition to establish boundaries for $R_{V}$

$$
\sqrt{\left\langle r_{n}^{2}\right\rangle} \leq \sqrt{\left\langle r_{o p}^{2}\right\rangle} \leq \sqrt{\left\langle r_{n}^{2}\right\rangle}+r_{f}
$$

For $\left\langle r_{n}^{2}\right\rangle$ the nuclear mean square charge radii of [9] were used. In order to obtain upper and lower boundaries for $R_{V}$, Eq. (2) was solved for different values of $R_{V}$ employing the associated initial parameter value for $a_{V}$.

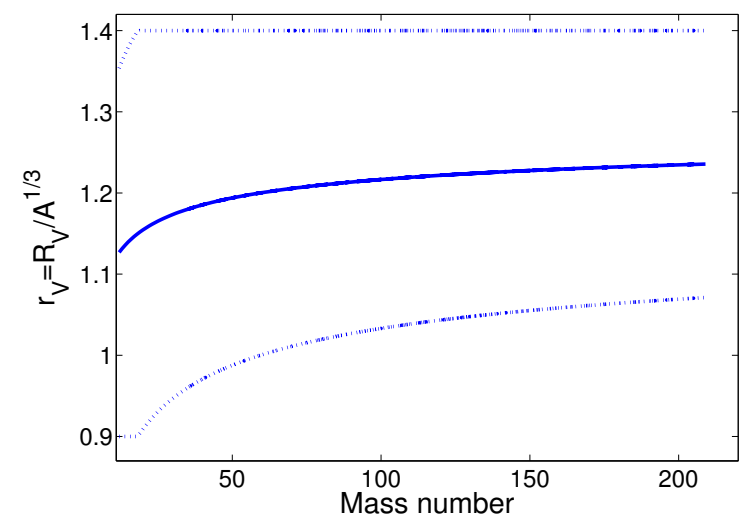

Fig. 2. Global values for the parameter $r_{V}$ and associated parameter boundaries obtained by Eqs. (2) and (3).

In Fig. 2, global values of $r_{V}=R_{V} / A^{1 / 3}$ and the associated parameter boundaries are displayed over the mass number $A$. Upper parameter boundaries of $r_{V}$ exceeding $1.4 \mathrm{fm}$ were considered to be unreasonably large and therefore set to $1.4 \mathrm{fm}$.

Parameter Boundaries for $d_{2}$ The parameter $d_{2}$ occurs in the energy-dependent part of the imaginary surface-central contribution $W_{D}(E)$ to the neutron optical potential of [4],

$$
W_{D}(E)=d_{1} \frac{\left(E-E_{f}\right)^{2}}{\left(E-E_{f}\right)^{2}+d_{3}^{2}} \exp \left\{-d_{2}\left(E-E_{f}\right)\right\},
$$

with the Fermi energy $E_{f}$. For this parameter, no physical argument was found to obtain reasonable parameter boundaries.

Fortunately, for some isotopes $d_{2}$-values are available for local and global parameter sets. These give a first indication of a physically reasonable parameter space for this specific isotope. Furthermore, following [5] we assumed that neighboring isotopes may show similar properties and thus parameters valid for one isotope might be reasonable for a neighboring nucleus as well.

In order to obtain parameter boundaries for a local parameter value $d_{2}(A)$ of a specific isotope with mass number $A$, we compared it with the local parameters for $d_{2}$ of isotopes within $\mathscr{C}=[A-10, A+10]$ and determined

$p_{d 2}(A)=\frac{1}{d_{2}(A)} \max \left(\max (\mathscr{C})-d_{2}(A), d_{2}(A)-\min (\mathscr{C})\right)$.

The upper and lower boundaries of $d_{2}(A)$ are obtained by

$$
d_{2}^{>}(A)=d_{2}(A)\left(1+p_{d 2}(A)\right)
$$

and

$$
d_{2}^{<}(A)=d_{2}(A)\left(1-p_{d 2}(A)\right)
$$

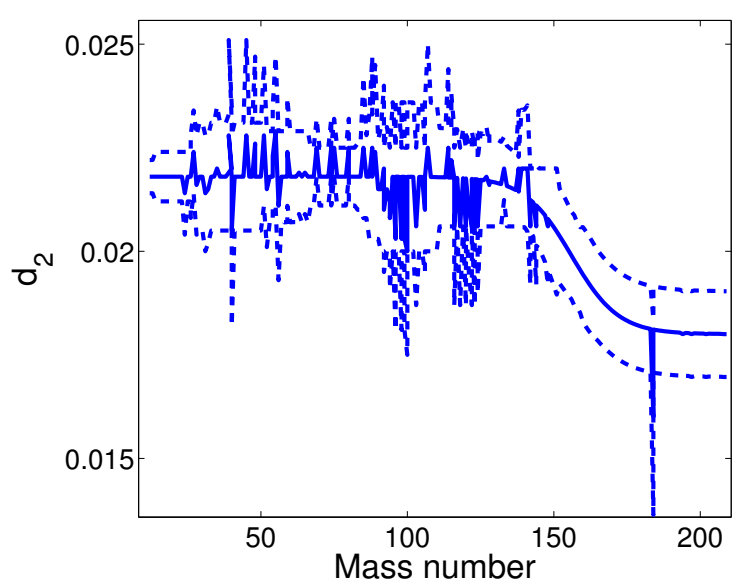

Fig. 3. Local values for the parameter $d_{2}$ and associated parameter boundaries.

The obtained parameter boundaries for $d_{2}$ are shown in Fig. 3. The unusual small value of $d_{2}$ for ${ }^{184} \mathrm{~W}$ was ignored during the analysis and treated separately. Above $A=162$, $p_{d 2}(A=162)$ was assumed for all $p_{d 2}$. The values $p_{d 2}$ obtained for the local $d_{2}$ were also used for the global $d_{2}$. All the local $d_{2}$ are also within the error bars of the global ones.

\subsection{Model Defect Covariance Matrices $A_{0}^{M D}$}

The model defect covariance matrices $A_{0}^{M D}$ should provide a measure for model deficiencies. In order to estimate $A_{0}^{M D}$, we used the 'scaling procedure' first proposed in [1] and refined in [2]. This phenomenological algorithm relies on the comparison of experimental cross sections $\sigma_{e}$ with model cross sections $\sigma_{t h}$ and implicitly assumes that the model reproduces experimental data of different isotopes in similar quality. Experimental data of different isotopes $n$ are 
employed. However, experimental data of the isotope to be calculated are not considered to avoid double counting. To put it in a nutshell, we define an energy-dependent scaling factor $\left\langle D_{n}^{c}\left(E_{m}\right)\right\rangle$ for each isotope $n$ and reaction channel $c$

$$
\left\langle D_{n}^{c}\left(E_{m}\right)\right\rangle=\sum_{j \in E_{\text {bin }}\left(E_{m}\right)} w_{j}^{\left(c, E_{m}, n\right)} \frac{\sigma_{e}^{(c, n)}\left(E_{j}\right)}{\sigma_{t h}^{(c, n)}\left(E_{j}\right)},
$$

and an energy-independent scaling factor $D^{c}$,

$$
D^{c}=\frac{1}{\sum_{n=1}^{N(c)}} \sum_{n=1}^{N(c)} \sum_{m=1}^{M} w_{m}^{(c, n)} \sqrt{K_{n}}\left\langle D_{n}^{c}\left(E_{m}\right)\right\rangle,
$$

which is weighted with the number of experimental data points $K_{n}$ for isotope $n$. $N(c)$ gives the total number of isotopes per reaction channel $c$ for which experimental data are available. The weights $w_{j}^{\left(c, E_{m}, n\right)}$ and $w_{m}^{(c, n)}$ are defined such that the largest $\sigma_{t h}$ either in energy bin $m$ or of the whole reaction channel $c$ respectively has the largest weight

The model defect covariance matrix is estimated by

$$
\begin{aligned}
& A_{0}^{M D}\left(E_{m}, E_{m^{\prime}}\right)=\frac{\sigma_{t h}^{c}\left(E_{m}\right) \sigma_{t h}^{c}\left(E_{m^{\prime}}\right)}{\tau^{c c^{\prime}}\left(E_{m}, E_{m^{\prime}}\right)} \times \\
& \sum_{n=1}^{N(c)}\left\{\left\langle\Delta D_{n}^{c}\left(E_{m}\right), \Delta D_{n}^{c}\left(E_{m^{\prime}}\right)\right\rangle+\delta_{c c^{\prime}} g_{m m^{\prime}} \Delta \tilde{D}_{n}^{c c^{\prime}}\left(E_{m}, E_{m^{\prime}}\right)\right\}
\end{aligned}
$$

with

$$
\Delta D_{n}^{c}\left(E_{m}\right)=\left\langle D_{n}^{c}\left(E_{m}\right)\right\rangle-D^{c}
$$

and a term

$$
\begin{aligned}
\Delta \tilde{D}_{n}^{c c^{\prime}}\left(E_{m}, E_{m^{\prime}}\right) & =\sqrt{\left\langle D_{n}^{c}\left(E_{m}\right)^{2}\right\rangle-\left\langle D_{n}^{c}\left(E_{m}\right)\right\rangle^{2}} \\
& \times \sqrt{\left\langle D_{n}^{c^{\prime}}\left(E_{m^{\prime}}\right)^{2}\right\rangle-\left\langle D_{n}^{c^{\prime}}\left(E_{m^{\prime}}\right)\right\rangle^{2}},
\end{aligned}
$$

where the latter simulates the systematic fluctuations of $\sigma_{e}$. The function

$$
g_{m m^{\prime}}=\frac{1}{2 \pi \omega} \exp \left\{-\frac{\left(E_{m}-E_{m^{\prime}}\right)^{2}}{2 \omega^{2}}\right\}
$$

models the width of these systematic fluctuations. With $\omega=1.5 \mathrm{MeV}$, a small width was chosen. The normalization factor $\tau^{c c^{\prime}}$ is given by the root of the number of isotopes for which experimental data were used for the reaction channel $c$ and $c^{\prime}$ and energy bin $m$ and $m^{\prime}$.

The 'scaling procedure' supplies $A_{0}^{M D}$ only for energy regions where experimental data are available. For other energy regions, the extrapolation algorithm of [2] is employed.

All experimental cross sections from 1 to $150 \mathrm{MeV}$ for the channels given in Table 1 were extracted from the EXFOR database [6]. They were carefully preselected to exclude erroneous data and obvious outliers. In order to reduce the weight of missed erroneous data and isotopes behaving very differently from all other isotopes, all experimental data were employed to calculate $A_{0}^{M D}$ for one specific isotope except the experimental data of the isotope itself. This was done in order to avoid double-counting in later evaluations. However, it makes no difference for the $A_{0}^{M D}$ whether the data of the considered isotope is used or not. This is not surprising due to the huge amount of experimental data involved.

\subsection{The Complete Prior}

The prior cross sections $\sigma_{0}^{c}$ for a channel $c$ are obtained by rescaling $\sigma_{t h}$ of Section 2.1 with the scaling factor of Eq. (4),

$$
\sigma_{0}^{c}=\sigma_{t h}^{c} D^{c}
$$

The prior covariance matrices $A_{0}$ are obtained according to [2] by

$$
A_{0}^{c c^{\prime}}\left(E_{m}, E_{m^{\prime}}\right)=D^{c} A_{0, m m^{\prime}}^{P U, c c^{\prime}} D^{c^{\prime}}+A_{0, m m^{\prime}}^{M D, c c^{\prime}} .
$$

Before combining $A_{0}^{P U}$ and $A_{0}^{M D}$, the covariance matrices are regularized by the algorithms described in [10] in order to restore positive semi-definiteness. Positive semi-definiteness of prior covariance matrices is violated due to numerical instabilities in the parameter uncertainties and due to fluctuating experimental data used for estimating model deficiencies. If $A_{0}^{P U}$ and $A_{0}^{M D}$ are positive semi-definite, the prior covariance matrix $A_{0}$ is also positive semi-definite.

\section{Example Evaluation with ${ }^{55} \mathrm{Mn}$ Prior}

In order to show the feasibility of the prior structural material library, we performed a test evaluation with local and global TALYS 1.0 prior data sets for ${ }^{55} \mathrm{Mn}$. The experimental data were inserted 'quasi blind' into the prior knowledge and no special measures were taken to improve the evaluated data.

This isotope was already evaluated with essentially the same theoretical procedures. In the previous evaluation [10] great care was taken to define a best-fitting initial parameter set specifically for this isotope, and the parameter boundaries for $A_{0}^{P U}$ were carefully established and revisited to give a consistent evaluation output.

As TALYS1.0 [4] was used for [10], we employ in this example evaluation also only the prior data for TALYS1.0. In the evaluation of [10], also the $(n, 4 n),(n, t)$ and $(n, p \alpha)$ reaction channels were evaluated using available experimental data of EXFOR [6]. These three reaction channels are not considered in the library and hence a new evaluation was undertaken starting from the prior of [10]. In this evaluation, the same experimental data were included as in the TALYS1.0 priors by means of the Full Bayesian Evaluation Technique, namely: Total, elastic, inelastic, $(n, 2 n)$, $(n, p)$ and $(n, \alpha)$ cross sections. The thus evaluated cross sections starting from the prior of [10], which are studied in this proceeding, do not differ visibly from the corresponding evaluated data of [10].

The cross sections of the global TALYS1.0 prior data set correspond well to the experimental cross sections up to $25 \mathrm{MeV}$, above they show systematic deviations, see Fig. 4. The corresponding prior uncertainties provide error bands which include all experimental data, although these uncertainties were established on global considerations and not specifically for this isotope. The different parameter space of the global TALYS1.0 prior and the prior of [10] can be clearly seen in the larger prior uncertainties of the global TALYS1.0 prior.

Using this prior in an evaluation provides evaluated cross sections which are in fair agreement with experimental data even for energies above $25 \mathrm{MeV}$. However, the obtained error bands are larger than those obtained using the 


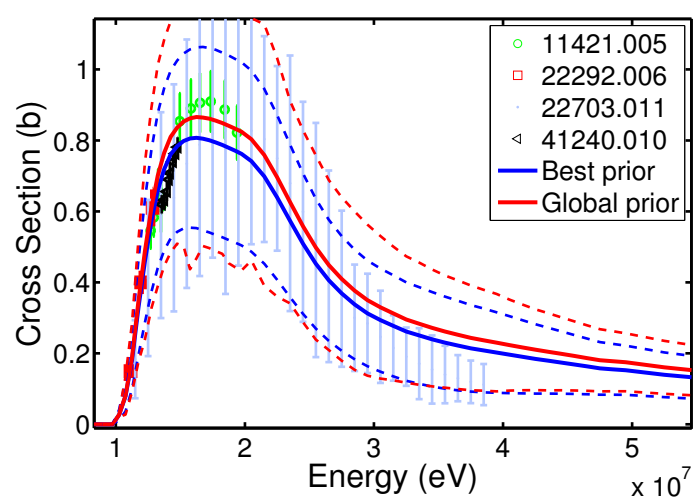

Fig. 4. Global TALYS1.0 prior cross sections (full red line) and uncertainties (red dashed lines) for the ${ }^{55} \mathrm{Mn}(n, 2 n)$ reaction channel in comparison to prior data of [10] (blue lines) and experimental data used in the evaluations.

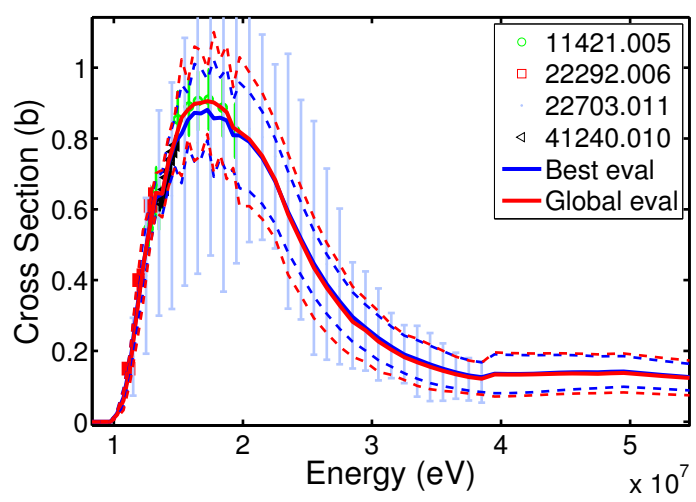

Fig. 5. Evaluated cross sections (full lines) and error bars (dashed lines) of ${ }^{55} \mathrm{Mn}(n, 2 n)$. The red data were obtained with the global TALYS1.0 prior and the blue data were obtained with the prior of [10].

prior of [10]. This reflects the difference in the parameter space which was restricted specifically for ${ }^{55} \mathrm{Mn}$ in [10], while the current prior was generated from a global consideration. In the energy range with accurately known experimental data the relative errors of the two evaluations are of the same size. Above $50 \mathrm{MeV}$, the evaluated relative errors of the global TALYS1.0 prior are $10 \%$ higher than those evaluated with the prior of [10]. However in this energy range the cross sections are already quite small and no major impact on derived quantities is expected. The covariance matrix evaluated with the global TALYS1.0 prior is similar to that using the prior of [10], see Figs. 7 and 8. The evaluated covariance matrix of the global TALYS1.0 prior differs visibly from the corresponding prior covariance matrix in the energy range of the experiments and shows similar properties above (see Fig. 6).

The evaluated $(n, 2 n)$ cross sections corresponding to the local TALYS1.0 data set also agree well with those calculated with the prior of [10] and the included experimental data, see Fig. 9. The evaluated cross sections of the local and the global data set show slightly different behavior between 17 and $27 \mathrm{MeV}$. This is caused by the different initial parameterization of the cross sections. This differences amounts to maximally $13.42 \%$ (mean deviation of $3.24 \%$ in this energy region) and both evaluated

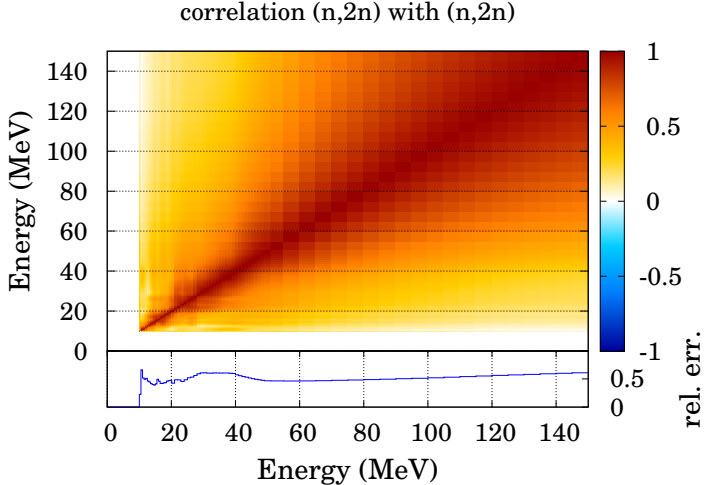

Fig. 6. Global TALYS1.0 prior correlation matrix and relative errors (box below) for ${ }^{55} \mathrm{Mn}(n, 2 n ; n, 2 n)$.

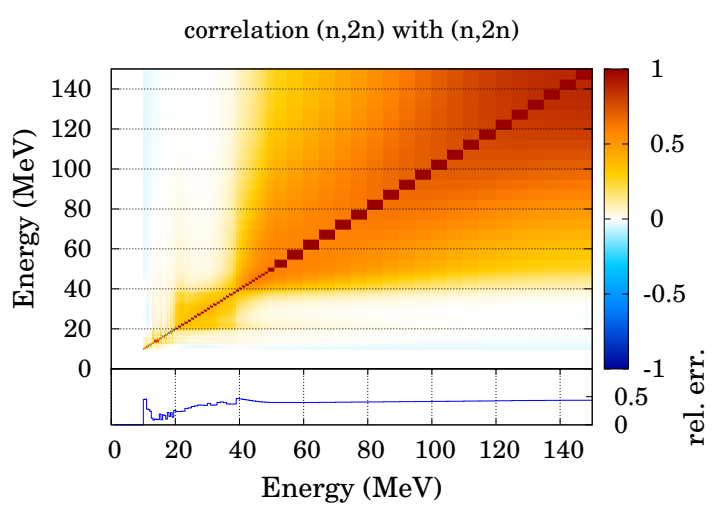

Fig. 7. Evaluated ${ }^{55} \mathrm{Mn}(n, 2 n)$ correlation matrix and relative errors (box below) for the global TALYS1.0 prior.

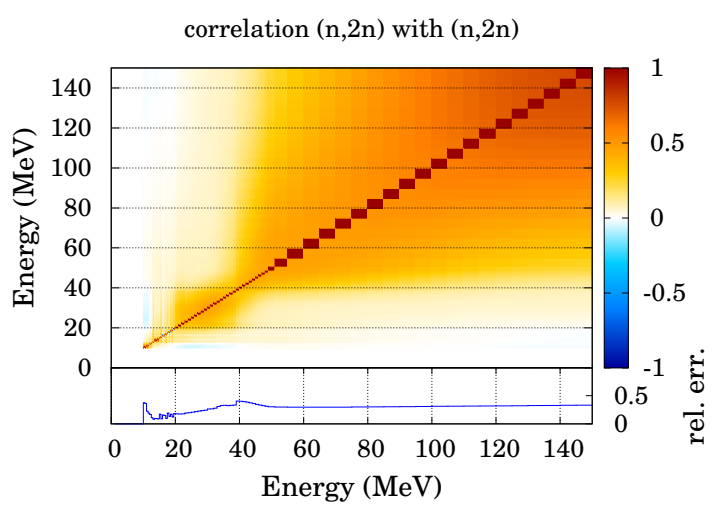

Fig. 8. Evaluated ${ }^{55} \mathrm{Mn}(n, 2 n)$ correlation matrix and relative errors (box below) for the prior of [10].

cross sections lie within the errorbars of the experimental data included in the evaluation. Especially, the agreement for the evaluated $(n, 2 n)$ cross sections beyond $25 \mathrm{MeV}$ with the experiment should be remarked because they have been all obtained from priors deviating systematically from the experiment at these energies. This 'healing of data' via evaluation is only possible if a proper $A_{0}^{M D}$ is included in the prior covariance matrix [11]. The evaluated covariance matrix of the local TALYS1.0 prior is very similar to the global TALYS1.0 one.

The 'least beautiful' evaluated cross sections were those obtained for the $(n, \alpha)$ reaction channel. The evaluated cross sections show kinks at 12 and $20 \mathrm{MeV}$. At $12 \mathrm{MeV}$, a transition between two different experimental data sets takes 


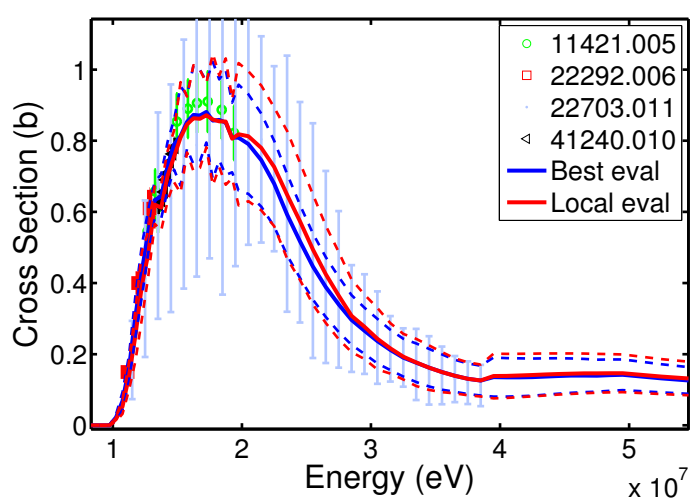

Fig. 9. Evaluated cross sections (full lines) and errorbars (dashed lines) of ${ }^{55} \mathrm{Mn}(n, 2 n)$. The red data were obtained with the local TALYS1.0 prior and the blue data were obtained with the prior of [10].

place while at $20 \mathrm{MeV}$ the experimental information ends. These kinks occur both in the global TALYS1.0 prior and in the evaluation starting from the prior of [10], although the kink at $12 \mathrm{MeV}$ is less pronounced with the prior of [10]. Both evaluations describe the experimental data well within the uncertainties. The kinks are caused by the weak prior correlations of the associated energy range and reflect at $12 \mathrm{MeV}$ the slightly diverging experimental data, Fig. 11. In order to obtain a smooth curve as expected from model data, smoothing algorithms can be applied.

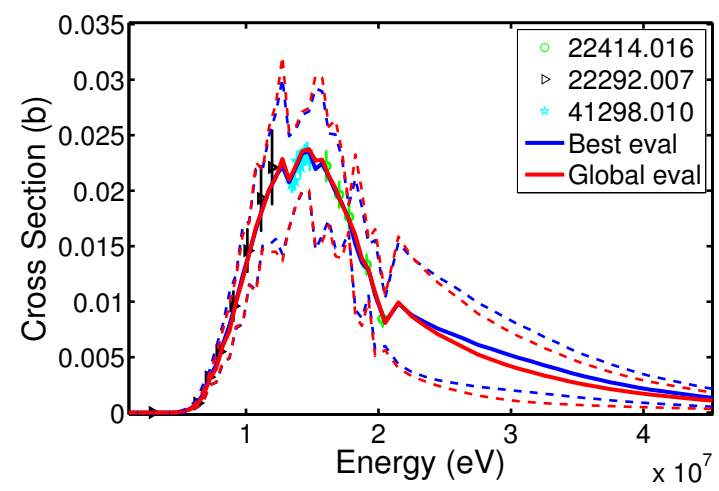

Fig. 10. Evaluated cross sections (full lines) and errorbars (dashed lines) of ${ }^{55} \mathrm{Mn}(n, \alpha)$. The red data were obtained with the global TALYS1.0 prior and the blue data were obtained with the prior of [10].

The evaluation starting from a prior specifically made for ${ }^{55} \mathrm{Mn}[10]$ and the 'blind evaluation' using global and local TALYS1.0 priors produced similar cross sections as well as covariance matrices when the same experimental data are entered into the evaluation process. In general, the evaluated errors of the evaluation with the global and local priors were larger than those evaluated with the prior of [10], which can be easily explained by the global construction of the former. This is a first indication that the prior structural material library might be feasible for future evaluations.

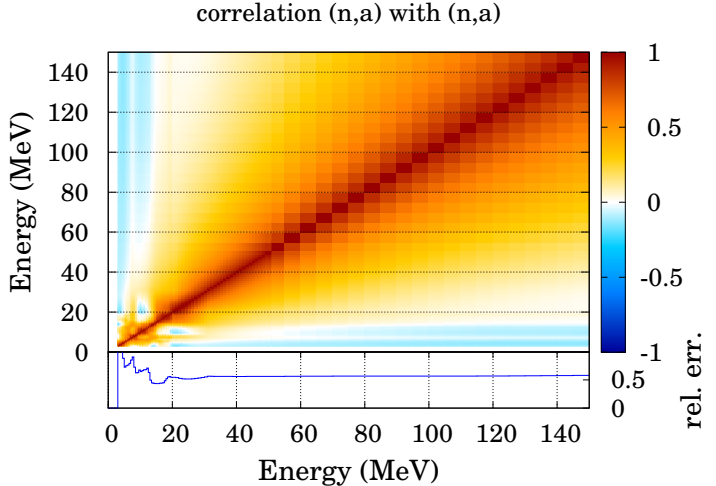

Fig. 11. Global TALYS1.0 prior correlation matrix and relative errors (box below) for ${ }^{55} \mathrm{Mn}(n, \alpha)$.

\section{Summary and Outlook}

A systematic generation of prior cross sections and covariance matrices for 276 isotopes relevant for structural materials has been performed. This prior knowledge can be employed as a fair starting point for future evaluations and reduces them effectively to the analysis of experimental data. The priors contain nine frequently measured channels and cover the energy range from 1 to $150 \mathrm{MeV}$. The priors are supplied in ENDF-6 format, providing $\mathrm{MF}=3$ and $\mathrm{MF}=33$ files. Additionally a $\mathrm{MF}=1$ file is supplied with general information regarding the specific isotope. The local and global optical potential parameters of [5] and the default parameters implemented in TALYS1.0 and TALYS1.2 [4] were employed to calculate the prior cross sections. The prior covariance matrices contain contributions from parameter uncertainties and model deficiencies. In order to estimate the former, parameter boundaries for all important parameters were carefully determined for each isotope in global studies. As examples we described the determination of boundaries for two parameters, one on the basis of physics arguments and one by empirical considerations. For the estimation of the model deficiencies, the scaling procedure of $[1,2]$ was employed and a wealth of experimental data throughout the periodic table was selected.

In order to show the feasibility of the library, a first 'blind' evaluation with a ${ }^{55} \mathrm{Mn}$ local and global TALYS1.0 prior of this library was performed. It corresponds well to an evaluation undertaken with the prior of [10] which was specifically established for this isotope. For all evaluations, the same experimental data were included and the same statistical method was employed. This indicates that the generated priors might provide a reasonable starting point for evaluations. However, further tests are still required.

At the moment, all necessary data are calculated and the assembly of the library is in progress. Investigations are undertaken to include further ENDF-6 files necessary for evaluators, e.g. $\mathrm{MF}=2$ and $\mathrm{MF}=4$ files. By end of June 2012, the testing and correcting phase will be finished and afterwards, the library will be available on the webpage www.geneus.eu.

This work was partly supported by the EURATOM project ANDES. The views and opinions expressed herein do not reflect necessarily those of the European Commission. The computational 
results presented have been achieved in part using the Vienna Scientific Cluster (VSC).

\section{References}

1. H. Leeb, D. Neudecker, Th. Srdinko, Nucl. Data Sheets 109, (2008) 2762

2. H. Leeb, St. Gundacker, D. Neudecker, Th. Srdinko, V. Wildpaner, Proceedings of Final Scientific EFNUDAT Workshop (Cern, Geneva 2010) 55

3. A. Trkov, R. Capote, E.Sh. Soukhovitskii, L.C. Leal, I. Kodeli, D.W. Muir, Nucl. Data Sheets 112 No. 12, (2011) 3098

4. A.J. Koning, S. Hilaire, M.C. Duijvestijn, Proceedings of the International Conference on Nuclear Data for Science and Technology ( EDP Sciences, Nice 2008) 211

5. A.J. Koning, J.P. Delaroche, Nucl. Phys. A 713, (2003) 231

6. IAEA Nuclear Data Section, EXFOR Formats Description for Users (IAEA-NDS-206, Vienna 2008)

7. H. Leeb, St. Gundacker, D. Neudecker, Th. Srdinko, V. Wildpaner, J. Korean Phys. Soc. 59 No. 2, (2011) 959

8. D. Neudecker, St. Gundacker, Th. Srdinko, V. Wildpaner, H. Leeb, Proceedings of Final Scientific EFNUDAT Workshop (Cern, Geneva 2010) 49

9. I. Angeli, Table of nuclear root mean square charge radii (INDC(HUN)-033, Hungary 1999)

10. V. Wildpaner, D. Neudecker, Th. Srdinko, H. Leeb, (these proceedings)

11. D. Neudecker, St. Gundacker, Th. Srdinko, V. Wildpaner, H. Leeb, J. Korean Phys. Soc. 59 No. 2, (2011) 1272 